\title{
Garlik: Semantic Technology for the Consumer
}

\author{
Nigel Shadbolt \\ CTO Garlik Ltd \\ Richmond \\ UK \\ School of Electronics and Computer Science \\ University of Southampton \\ Highfield \\ Southampton, SO17 1BJ \\ United Kingdom \\ http://users.ecs.soton.ac.uk/nrs/
}

In under a decade the internet has changed our lives. Now we can shop, bank, date, research, learn and communicate online and every time we do we leave behind a trail of personal information. Organisations have a wealth of structured information about individuals on large numbers of databases. What does the intersection of this information mean for the individual? How much of your personal data is out there and more importantly, just who has access to it? As stories of identity theft and online fraud fill the media internet users are becoming increasingly nervous about their online data security. Also what opportunities arise for individuals to exploit this information for their own benefit?

Garlik was formed to give individuals and their families real power over the use of their personal information in the digital world. Garlik's technology base has exploited and extended results from research on the Semantic Web. It has built the world's largest, SPARQL compliant, native format, RDF triple store. The store is implemented on a low-cost network cluster with over 100 servers supporting a $24 \times 7$ operation. Garlik has built semantically informed search and harvesting technology and has used industrial strength language engineering technologies across many millions of people-centric Web pages. Methods have been developed for extracting information from structured and semi structured databases. All of this information is organised against a people-centric ontology with facilities to integrate these various fragments.

Garlik has received two substantial rounds of venture capital funding (as of March 2008), has established an active user base of tens of thousands of individuals, and is adding paying customers at an increasing rate. This talk reviews the consumer need, describes the technology and engineering, and discusses the lessons we can draw about the challenges of deploying Semantic Technologies. 